\title{
Índice de autores
}

\begin{tabular}{|c|c|c|c|c|c|}
\hline \multirow{2}{*}{\multicolumn{2}{|c|}{$A$}} & Gómez Buela, Inmaculada & 77,91 & Pérez Álvarez, Roberto & $81,82,93$ \\
\hline & & Gómez Gigirey, Adriana & 89,93 & Perez Carral, Oscar & 91 \\
\hline Abreu, Diana & 88 & González González, Lucía & $75,77,78,79,81$ & Pérez Carral, Verónica & $85,87,91$ \\
\hline Alonso, Ana & 76 & & $82,83,84,86,87$ & Pérez Iglesias, Almudena & 85,91 \\
\hline Alonso Herrero, Ana & $81,83,86$ & & $89,90,91,92,93$ & Pérez Pombo, Serafín & 85 \\
\hline Alonso, $\mathrm{M}$ & 78,89 & González Piñeiro, Ana & 85 & Pérez Rodríguez, María Teresa & $75,76,77,78,81$ \\
\hline Alonso Parada, María & $\begin{array}{l}75,76,79,84,85 \\
90,92\end{array}$ & $\begin{array}{l}\text { González Quintela, Arturo } \\
\text { González Vázquez, Elvira }\end{array}$ & $\begin{array}{l}82 \\
85,87\end{array}$ & & $\begin{array}{l}83,84,86,89,91, \\
92\end{array}$ \\
\hline Álvarez Fernández, Maximiliano & 77 & Guitián, Ramón & 84 & Pernas Souto, Berta & 87,89 \\
\hline Álvarez, M & 83 & & & Piñeiro Fernandez, Juan Carlos & 88 \\
\hline Álvarez Pequeño, Leticia & 75,84 & l & & Portela, Daniel & 78,84 \\
\hline Álvarez Pérez, Joaquin & $81,82,93$ & Jñiouez Vázauez lria & 7688 & Porto Pérez, Ana Belkis & 78,87 \\
\hline Álvarez Rodríguez, Elena & 75,85 & innguel valquel, illa & 10,80 & & \\
\hline Alves, Eide Diana & 84,85 & 1 & & K & \\
\hline Angell Valdés, Sussette & 80 & U & & Rabuñal Rey, Ramón & 76,88 \\
\hline Anibarro, Luis & 84,85 & Jorge Sánchez, Ramón & 89 & Ramos, Vicente & $\begin{array}{l}10,00 \\
89\end{array}$ \\
\hline Argibay Filgueira, Ana Belén & $\begin{array}{l}75,76,77,78,81 \\
82,83,84,86,89\end{array}$ & & & Regueiro Dominguez, Alejandro & 88 \\
\hline & $90,91,92$ & $L$ & & Rey González, Lara & 87 \\
\hline Armas Castro, Julio & 80 & Lijó Carballeda, Cristina & 89,91 & Ríos, Mónica & 84,85 \\
\hline & & Lito, Pedro & 88 & Rivas Costa, Gonzalo & 77,89 \\
\hline$B$ & & Longueira, Rebeca & 76 & Rivera, Alberto & $75,79,87,89$ \\
\hline Ballesteros Ballesteros, Rosa Maria & 88 & López Miragaya, Isabel & 86 & Rivera Fraga, Carmen & 93 \\
\hline Barbagelata López, Cristina & $77,86,89,90,91$ & López Otero, José Luis & $81,82,93$ & Rivera Gallego, Alberto J. & $75,77,81,83,85$, \\
\hline Boceta Osuna, Jaime & & Lorenzo Castro, Rut & $77,78,79,81,82$ & Rodil Rodil, Viviana & $\begin{array}{l}90,92 \\
82,93\end{array}$ \\
\hline Buño Ramilo, Beatriz & 79 & & $83,84,85,86,89$, & Rodríguez Álvarez, Ana Paula & $85,87,91$ \\
\hline C & & & & Rodríguez, Ana & 79,87 \\
\hline U & & & & Rodríguez Cordero, Marte & 82 \\
\hline Caínzos Romero, Tamara & $75,77,79,80,89$ & $M$ & & Rodríguez da Silva, Alfredo & 76 \\
\hline & $\begin{array}{l}75,71,19,80,89 \\
9091\end{array}$ & IVI & & Rodríguez Gómez, Ana & 76,81 \\
\hline Caride López, Gloria & 93 & Machado Prieto, Begoña & 82,93 & Rodriguez Gómez, Ana María & $76,78,81,82,83$, \\
\hline Casal Iglesias, Luis & $\begin{array}{l}93 \\
89\end{array}$ & Marnotes González, Javier & 87 & & $86,89,90,91,92$ \\
\hline Casal, Moisés & 77 & Martínez, Ana & 79,87 & Rodríguez-Gómez, Ana María & 77 \\
\hline Castelo, Laura & 89 & Martínez Lamas, Lucía & $76,77,91$ & Royo Aguado, José Luis & 78 \\
\hline Chamorro Fernández, Antonio J. & $85,87,91$ & Martínez, Lucía & 83,86 & Rubal Bran, David & 76,88 \\
\hline Cubillo, Severina & 87 & Martínez, Mar & 87 & S & \\
\hline & & Martínez Vázquez, César & 78.76 .77 .78 .79 & $\mathcal{J}$ & \\
\hline U & & martirive vacquec, grour & $81,83,84,85,86$ & Saavedra Martínez, Pablo & 76 \\
\hline De La Iglesia, Fernando & 89 & & $89,90,91,92$ & Sanchez Trigo, Sabela & 90 \\
\hline De Toro Santos, José Manuel & 85,91 & Martínez Vidal, Ana Isabel & $76,77,78,81,82$ & Sardina Ferreiro, Raquel & 77,91 \\
\hline Díaz Peromingo, José Antonio & 82 & & $83,86,89,90,91$ & $\begin{array}{l}\text { Seco Hernandez, Elena } \\
\text { Serrano Joaquin }\end{array}$ & $\begin{array}{l}85,87,91 \\
89\end{array}$ \\
\hline Diéguez, Julia & 84,85 & & 92 & Sesma Sánchez, Pascual & $75,77,78,79,80$ \\
\hline Dopico Lopez, Alfonso & 81,89 & Matesanz Fernández, María & 76,88 & & $82,83,84,87,89$ \\
\hline Doutel Haghighi, Eduardo & 88 & Maure Noia, Brenda & 86 & & $85,89,90,91,92$ \\
\hline Duarte Bermudez, Blanca Paulina & 90 & $\begin{array}{l}\text { Mella Pérez, Carmen } \\
\text { Miguélez Rodríquez, Daniela }\end{array}$ & $\begin{array}{l}77,86,89,90,91 \\
82,93\end{array}$ & & 93 \\
\hline$F$ & & Miralles, Celia & 76 & Sousa Domínguez, Adrián & $76,77,78,79,81$ \\
\hline & & Montero Teijeiro, Carmen & 78,87 & & $82,83,84,86,89$, \\
\hline Fernández, Alberto & 84,85 & Monte Secades, Rafael & 76,88 & & 92 \\
\hline Fernández Bouza, Elena & $77,86,89,90,91$ & Montes Santiago, Julio & 82,93 & $T$ & \\
\hline Fernández Calvo, Laura & 80 & Mosquera Pena, Marcelino & 93 & l & \\
\hline Fernández Fernández, Francisco J & 75,80 & Mouronte Sánchez, Camino & 87 & Trigás Ferrín, María & $75,79,80$ \\
\hline Fernández Regal, Inés & $85,87,91$ & & & & \\
\hline Fernández Rial, Alvaro & 93 & IV & & V & \\
\hline Fernández Victoria, Rebeca & 79 & Nicolas, Ricardo & 89 & & \\
\hline Ferreira González, Lucía & $75,79,80$ & Nodar Germiñas, Andrés & $77,78,83,84,86$ & $\begin{array}{l}\text { Valcarcel Garcla, Marla Angeles } \\
\text { Vaqueiro Rodríguez, Inés }\end{array}$ & $\begin{array}{l}82 \\
76\end{array}$ \\
\hline Freire Dapena, María del Carmen & 90 & & 89,92 & Vázquez Triñanes, Caritina & $75,77,78,79,81$ \\
\hline $\begin{array}{l}\text { Freire Dapena, Mayka } \\
\text { Freire }\end{array}$ & $\begin{array}{l}75,77,85,87,90 \\
89\end{array}$ & Núñez Calvo, Luisa & 87 & & $82,83,84,87,89$ \\
\hline $\begin{array}{l}\text { Frere, IV } \\
\text { Freire, Ramón }\end{array}$ & $\begin{array}{l}89 \\
87\end{array}$ & Núñez, Manuel Jesús & 84,85 & & $90,91,92$ \\
\hline & & & & Vázquez Vázquez, Begoña & $77,86,89,90,91$ \\
\hline$G$ & & & & Ventura Valcarcel, Pablo & 88 \\
\hline & & Ocampo Antonio & 76 & Vicente, Leopoldina & 88 \\
\hline García, Carlos & 87 & 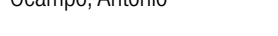 & & Viejo, Miguel Ángel & 84,85 \\
\hline García del Río Beneyto, Rocio & 80 & $D$ & & Vilariño Maneiro, Laura & $75,79,80,89,91$ \\
\hline García, Juan Carlos & 84,85 & $P$ & & Villaverde Álvarez, Iria & $75,76,79,81,82$ \\
\hline García Martín, José Carlos & 78 & Pellicer, Carlos & 89 & & $83,84,85,87,90$, \\
\hline Gascó Sogorb, María Jesús & 80 & Penado, Susana & 89 & & 92 \\
\hline Giménez Fernández, Blas & 80 & 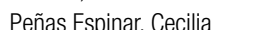 & & Villaverde, I & 78,89 \\
\hline
\end{tabular}

